\title{
A Memetic Genetic Algorithm for the Vertex p-center Problem
}

Wayne Pullan

w.pullan@griffith.edu.au

School of Information and Communication Technology, Griffith University,

Gold Coast, QLD, Australia

\begin{abstract}
The $p$-center problem is one of choosing $p$ facilities from a set of candidates to satisfy the demands of $n$ clients in order to minimize the maximum cost between a client and the facility to which it is assigned. In this article, PBS, a population based meta-heuristic for the $p$-center problem, is described. PBS is a genetic algorithm based meta-heuristic that uses phenotype crossover and directed mutation operators to generate new starting points for a local search. For larger $p$-center instances, PBS is able to effectively utilize a number of computer processors. It is shown empirically that PBS has comparable performance to state-of-the-art exact and approximate algorithms for a range of $p$ center benchmark instances.
\end{abstract}

Keywords

Memetic genetic algorithms, combinatorial optimization.

\section{Introduction}

The $p$-center problem calls for finding the $p$ facilities that minimize the maximum cost of servicing each of $n$ clients, where the pairwise cost of servicing each client from all facilities is given. Each client is only serviced by a single facility and services to clients are not combined. Formally, the $p$-center problem can be stated as (Mladenović et al., 2003): Let $V=\left\{v_{1}, v_{2}, \ldots, v_{n}\right\}$ be a set of $n$ potential locations for facilities and $U=\left\{u_{1}, u_{2}, \ldots, u_{m}\right\}$ a set of $m$ users. The distance between (or cost incurred for) each user-facility pair $\left(u_{i}, v_{j}\right)$ is given as $d_{i j}=d\left(u_{i}, v_{j}\right)$. The $p$-center problem is to find $S \subseteq V$ of size $p$ such that $f(S)=\max \left\{\min d\left(u_{i}, v_{j}\right)\right\}, u_{i} \in U, v_{j} \in S$ is minimized. The vertex $p$-center problem addressed in this study is where $V=U$ (that is, all vertices can be either a facility or a user) and is simply referred to as the $p$-center problem (PC) in the remainder of this article.

PC is a prominent combinatorial optimization problem from location science (Tansel et al., 1983) and has been shown to be NP-hard (Kariv and Hakimi, 1979). Exact techniques for the $p$-center problem are typically based on the fact that the optimal value for $f(S)$ can be determined by solving a sequence of different, auxillary, subproblems. Minieka (1970) proposed an algorithm that involved solving a finite series of set covering problems to identify the minimum threshold distance at which all customers are covered using $p$ facilities. Based on this algorithm, Daskin (1995) developed an algorithm that found the minimum threshold distance using a bisection method which systematically reduced the gap between the upper and lower bounds of the optimal solution. This algorithm was further developed in Daskin (2000) where a maximum 
set covering subproblem was formulated and solved using Langrangian relaxation. Ilhan and Pinar (2001) proposed a method that first finds a lower bound to the optimal solution for the problem by solving a series of feasibility problems based on a linear programming formulation and then, using an integer programming technique, uses feasibility problems to check for feasible solutions. The exact algorithm of Elloumi et al. (2004) solved the set covering subproblems using a greedy heuristic and an integer programming formulation of the subproblem. More recently, Al-Khedhairi and Salhi (2005) developed modifications to both the Daskin (1995) and Ilhan and Pinar (2001) algorithms that improved their efficiency.

Unlike the other basic discrete location problem, the $p$-median problem (Resende and Werneck, 2004; Cranic et al., 2004; Taillard, 2003; Hansen et al., 2001), few heuristic approaches to solving the $p$-center problem have been proposed. Initially, heuristics (Hochbaum and Shmoys, 1985; Martinich, 1988; Plesnik, 1987) exploited the close relationship between the $p$-center problem and the dominating set problem. After a gap of 10 years the next, and most recent, meta-heuristic for the $p$-center problem was proposed by Mladenović et al. (2003) where local search was combined with the meta-heuristics: Multi-Start, Tabu Search, and Variable Neighbourhood Search (VNS) and compared with three classical heuristics Greedy, Alternate, and Interchange (the most common heuristics used for the $p$-median problem) in addition to the Binary Search heuristic (Hochbaum and Shmoys, 1985). The conclusion reached was that, overall, the combination of VNS with local search had the best performance of the heuristics examined. In Hassin et al. (2003), a local search strategy is proposed that is directed at combinatorial optimization problems with a min-max (or max-min) objective. Using this approach, solutions are compared lexicographically rather than by their worst coordinate and they applied this local search strategy to a number of small $p$-center problems. Previous theoretical studies of problems related to the $p$-center problem include Krumke (1995) and Cheng et al. (2007) while Scaparra et al. (2004) investigates the capacitated $p$-center problem.

This article describes a memetic genetic algorithm, dubbed PBS, for the $p$-center problem, which incorporates phenotype crossover and directed mutation operators, to generate new starting points for a local search. For larger $p$-center instances, PBS is able to effectively utilize a number of computer processors. Based on computational experiments, this study demonstrates that PBS has excellent performance with regard to robustness, quality of results, and computational requirements, on a range of PC benchmark instances, and hence represents an improvement in heuristic PC solving algorithms. Descriptive results on the behavior of the components of PBS are also presented that give insights into the roles of these components during the search.

The remainder of this article is structured as follows. The PBS algorithm is first described. Next, empirical performance results are presented that establish PBS as an effective meta-heuristic for solving the PC problem. This is followed by a more detailed investigation of the behavior of PBS and the factors determining its performance. Finally, the main contributions of this work are summarized, insights gained from this study are presented, and some directions are outlined for future research.

\section{The PBS Algorithm}

The PBS algorithm is based on the fundamental concept of augmenting a population based meta-heuristic with an iterative improvement local search. The goal of the metaheuristic is to generate a wide range of "good" starting points for the local search while the role of the local search is to effectively explore regions that are relatively close to these 
starting points. Key features of the PBS algorithm are now described using the following terminology and syntax: $G=(V, E)$-a directed or undirected graph, where $V$ is the set of all $n$ vertices and $E$ is the set of all possible edges; $S-p$-center solution $(S \subseteq V)$; $p$ required number of facilities in $S$; facility- $v \in S ; S_{c}-\max \left\{\min d\left(v_{i}, v_{j}\right)\right\}, v_{i} \in V, v_{j} \in S$, that is, the maximum cost of servicing vertices in $V$ from the facilities in $S$. Each vertex is serviced by a single facility and the cost of servicing all vertices from all facilities is predefined for each instance; $|S|$-the number of facilities currently in $S ; P$-PBS population of $p$-center solutions; $P_{i}$-a $p$-center solution within the population $P ; N_{v}-$ distance ordered list of vertices that are neighbors of vertex $v$ (note that $N_{v}[0]=v$ ); $N_{v k}$-a sublist containing the first $k$ elements of $N_{v}\left(=\left\{N_{v}[0], \ldots, N_{v}[k-1]\right\}\right)$; and, $N_{p}-$ the number of computer processors to be used by PBS.

As an overview and with reference to the high-level pseudo-code description of the PBS algorithm that follows, PBS uses a relatively small population $P$ of eight $p$-center solutions (initially, randomly generated $(\mathcal{C})$ and optimized using the local search $(\mathcal{L})$ ) to provide inputs to genetic mutation $\left(\mathcal{M}_{1}, \mathcal{M}_{2}\right)$ and crossover $\left(\mathcal{X}_{1}, \mathcal{X}_{2}\right)$ operators. The outputs from these operators subsequently become inputs to the local search which produces candidate solutions for addition to $P$. This process is repeated until either a target cost is attained or the maximum allowed number of generations is achieved.

\section{Algorithm PBS}

(Create population $P$ )

1. $\forall P_{i} \in P, P_{i} \leftarrow \mathcal{L}(\mathcal{C})$

2. while not finished

3.

(Perform each generation)

4.

5.

$$
\begin{aligned}
\forall P_{j} & \in P-P_{i} \\
& P \leftarrow P+\mathcal{L}\left(\mathcal{M}_{1}\left(P_{j}\right)\right) \\
& P \leftarrow P+\mathcal{L}\left(\mathcal{M}_{2}\left(\mathcal{X}_{1}\left(P_{i}, P_{j}\right)\right)\right) \\
& \left(S_{1}, S_{2}\right) \leftarrow \mathcal{X}\left(P_{i}, P_{j}\right) ; \\
& P \leftarrow P+\mathcal{L}\left(\mathcal{M}_{2}\left(S_{1}\right)\right)+\mathcal{L}\left(\mathcal{M}_{2}\left(S_{2}\right)\right)
\end{aligned}
$$

In more detail, at the commencement of PBS (Line 1), all the initial solutions for $P$ are created $(\mathcal{C})$ by setting each $P_{i}$ to a single vertex chosen uniformly at random from the given graph $G$. Then the local search $(\mathcal{L})$ is invoked to first increase the number of facilities in $P_{i}$ to $p$ after which an iterative improvement phase is entered and facility-vertex swaps are performed for some number of iterations. Once the initial population has been created, PBS repeatedly performs generations (Lines 2-8) during which crossovers $\left(\mathcal{X}_{1}, \mathcal{X}_{2}\right)$ between all possible combinations of $\left(P_{i}, P_{j}\right)$ and $|P|(|P|-1) \mathcal{M}_{1}$ mutations of each $P_{i} \in P$ are performed. In addition, $\mathcal{M}_{2}$ mutations are performed on all child solutions generated by the $\mathcal{X}_{2}$ crossover. Immediate updating of $P$ is performed whenever a lower cost $p$-center solution is found. At the completion of each crossover and mutation operation, child solutions $S$ are locally optimized using the local search $\mathcal{L}$ which, if $|S|=p$ iteratively removes a facility and replaces it with the vertex, the result is the maximum decrease/smallest increase in the cost of $S$. If $|S|<p$, then the local search simply adds the vertex, which results in the largest decrease in the cost of $S$. To maintain diversity in the population, no new $p$-center solution $S$ is added ( + ) to $P$ if it is close (in cost or facilities) to any $P_{i}$ in $P$.

PBS is able to execute in either a single processor mode, as described in the PBS algorithm above (referred to as PBS-1 in this article), or in a parallel processing mode (referred to as PBS- $x$ in this article, where $x$ is the number of computer processors 
utilized). PBS- $x$ implements the PBS algorithm using a simple master-slave model of parallel processing where the single master task controls the distribution of pairs of solutions from $P$ to each slave task and also the potential updating of the population $P$ when the slave tasks return the results from all crossover and mutation operators (and the subsequent local searches). That is, for PBS- $x$, all $\mathcal{C}, \mathcal{X}_{1}, \mathcal{X}_{2}, \mathcal{M}_{1}, \mathcal{M}_{2}$, and $\mathcal{L}$ operations are performed in parallel in the slave tasks, thus providing an $x$-way overlapping of the mutation, cross-over, and local search portions of the PBS algorithm. Communication between the master and each slave task and control of the slave tasks by the master task is performed using messages passed through the MPI message passing interface (http: //www-unix.mcs.anl.gov/mpi/). A copy of the code for PBS can be obtained by contacting the author.

\subsection{Mutation Operators $\mathcal{M}_{1}$ and $\mathcal{M}_{2}$}

Within PBS, two mutation operators exist. The first, $\mathcal{M}_{1}$, is a random mutation operator that takes a $p$-center solution $P_{i}$ and constructs a child solution $S$ by first randomly selecting some number $q$ in the range $p / 2, \ldots, p$ and then using $q$ randomly selected facilities from $P_{i}$ and $p-q$ randomly selecting vertices from $V-P_{i}$ to construct $S$. The local search is then invoked using $S$ as the starting point and the resulting $p$-center solution becomes a candidate for addition to $P$. The second mutation operator $\left(\mathcal{M}_{2}\right)$ is a directed mutation operator that, based on the assumption that facilities should not be "close" to each other, identifies the closest pair of facilities within $P_{i}$, deletes them from $P_{i}$, and then invokes local search to generate a new $p$-center solution which becomes a candidate to be added to $P$.

\subsection{Crossover Operators $\mathcal{X}_{1}$ and $\mathcal{X}_{2}$}

The specific crossover operators implemented within PBS are: $\mathcal{X}_{1}$, a random crossover operator which constructs a single child solution by randomly selecting $p$ facilities from the two parents; and $\mathcal{X}_{2}$, which first randomly selects two users and a random number $q \in[0.1, \ldots, 0.9]$. Let $d_{i 1}$ be the distance between facility $i$ and the first selected user and $d_{i 2}$ the distance between facility $i$ and the second selected user. The first child solution is constructed by combining all facilities $i$ in the first parent where $d_{i 1} / d_{i 2} \leq q$ with all facilities $j$ in the second parent where $d_{j 1} / d_{j 2}>q$. The second child solution is built by combining all facilities $i$ in the first parent where $d_{i 1} / d_{i 2}>q$ with all facilities $j$ in the second parent where $d_{j 1} / d_{j 2} \leq q$. For this crossover operator, if more than $p$ facilities are added to a child solution then the required number are randomly removed to reduce the total down to $p$. If less than $p$ facilities are present then the subsequent local search will add the required number to bring the child solution back to $p$ facilities. The motivation for this crossover operator is that, one of the primary functions of crossover genetic operators is the identification of "good" building blocks within parent solutions and the extraction of these into the children solutions. Clearly these good building blocks will tend to exist in the $p$-center problem as groups of facilities that are, physically, relatively close to each other and this physical relationship is not preserved within the $p$-center solution $S$. The $\mathcal{X}_{2}$ operator, which operates in the physical space, does create building blocks of physically close facilities whereas the $\mathcal{X}_{1}$ operator, which operates directly on the $S$ vector, does not. It should be noted that equivalent phenotype crossover operators have been very successfully applied to clustering problems in other domains where there is a distance dependent interaction between the elements of the problem [e.g., the structural optimization of atomic clusters (Pullan, 2005)]. 


\subsection{Local Search $\mathcal{L}$}

The local search component of PBS takes a $p$-center solution $S$ and, if $|S|<p$, adds facilities to $S$ by uniform randomly selecting a facility from $N_{w k}$ where $w$ is a vertex whose distance from the nearest facility is currently the maximum (i.e., $S_{c}$ ) and $k$ is the index in $N_{w}$ of the facility that currently services vertex $w$. Once $|S|=p$, the local search enters a swap phase where, for each iteration of the local search, a facility is removed from $S$ and replaced by a vertex that has not previously been swapped with that facility. Selection of the vertex to be added to $S$ is performed by evaluating, for each vertex in $N_{w k}$, the overall changes in $S_{c}$ by swapping this vertex with each facility in $S$. The actual vertex and facility to be swapped is that pair which provides the best decrease (or least increase) in $p$-center cost. To prevent the local search cycling around vertices, once a facility/vertex swap has been performed, that combination is no longer eligible for selection during the current invocation of the local search. The termination criteria for the local search is either $2 n$ iterations or no decrease in $S_{c}$ for $0.1(g+1) n$ iterations (where $g$ is the PBS generation count). Experiments, not reported in this article, were performed to determine the form of and values used in these termination criteria.

The local search was primarily designed to keep the time-complexity of the individual search steps minimal which is in keeping with the common intuition that, in the context of stochastic local search algorithms, it is often preferable to perform many relatively simple, but efficiently computable search steps rather than fewer complex search steps. As the local search function of PBS is the major computational component of PBS, an efficient algorithm is essential. The PBS data structures that allow the efficient implementation of local search are based on those used in the VNS meta-heuristic (Mladenović et al., 2003) (which in turn are based on those developed by Whitaker, 1983), and are as follows: $F_{v}^{0}$-facility which is closest to vertex $v ; D_{v}^{0}$ - distance from vertex $v$ to the closest facility; $F_{v}^{1}$-facility which is second closest to vertex $v ; D_{v}^{1}$-distance from vertex $v$ to the second closest facility. Using these data structures and the previously defined notation, the three key functions within local search are now described:

\section{Procedure Add_Facility $(f)$}

1. $\quad S_{c} \leftarrow 0$

2. $S \leftarrow S \cup f$

3. $\forall v \in V$

4. $\quad$ if $d_{f v}<D_{v}^{0}$

5. $\quad D_{v}^{1} \leftarrow D_{v}^{0} ; F_{v}^{1} \leftarrow F_{v}^{0} ; D_{v}^{0} \leftarrow d_{f v} ; F_{v}^{0} \leftarrow f$

6. $\quad$ else if $d_{f v}<D_{v}^{1}$

7. $\quad D_{v}^{1} \leftarrow d_{f v} ; F_{v}^{1} \leftarrow f$

8. $\quad$ if $D_{v}^{0}>S_{c}$

9. $\quad S_{c} \leftarrow D_{v}^{0}$

Procedure Add_Facility has worst-case complexity $\mathcal{O}(n)$ and adds facility $f$ to the $p$-center solution $S$, updates the data structures and determines the cost $S_{c}$ of $S$.

\section{Procedure Remove_Facility $(f)$}
1. $S_{c} \leftarrow 0$
2. $S \leftarrow S-f$
3. $\forall v \in V$
4. $\quad$ if $F_{v}^{0}=f$ 
5.

6.

$$
D_{v}^{0} \leftarrow D_{v}^{1} ; F_{v}^{0} \leftarrow F_{v}^{1} ;\left(D_{v}^{1}, F_{v}^{1}\right) \leftarrow \text { Find_Next }(v)
$$

7.

8. $\quad$ if $D_{v}^{0}>S_{c}$ else if $F_{v}^{1}=f$

$$
\text { 9. } \quad S_{c} \leftarrow D_{v}^{0}
$$

Procedure Remove_Facility removes facility $f$ from the $p$-center solution $S$, updates the data structures, and determines the cost $S_{c}$ of $S$. The function Find_Next $(v)$ scans $S$ to find the second closest facility to $v$ after $f$ has been removed from $S$. While Remove_Facility has worst-case complexity $\mathcal{O}(n p)$, from a practical point of view, it is extremely unlikely that this will occur as it would require facility $f$ to be either the closest or second closest facility to all $v \in V$. The other mitigating factor is that, as described below, Remove_Facility is used only once per iteration of the local search.

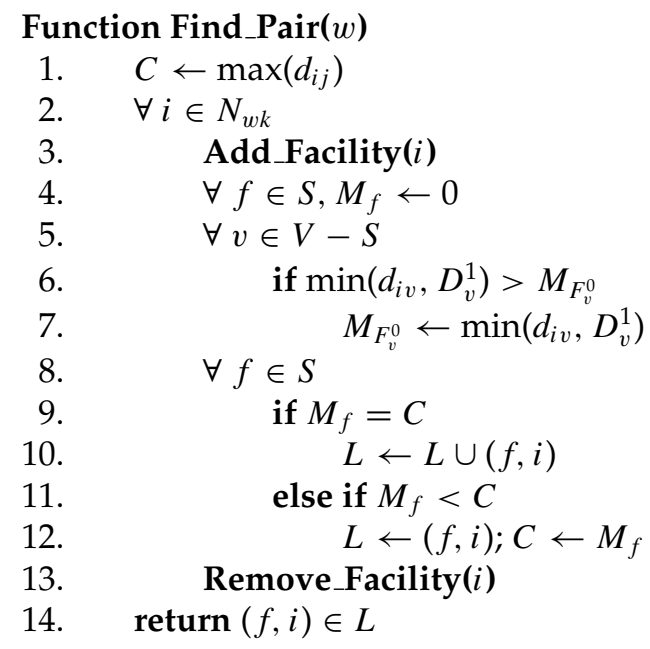

Function Find_Pair finds an optimal (facility, vertex) pair to swap. Clearly, to improve $S_{c}$, a vertex in $N_{w k}$, where $D_{w}^{0}=S_{c}$ and $k$ is the index of $F_{w}^{0}$ in $N_{w}$, must be added to $S$. Function Find_Pair evaluates, for all vertices in $N_{w k}$, the changes in $S_{c}$ caused by adding that vertex and removing, in turn, all existing facilities in $S$ (in the algorithm description for Find_Pair, $M_{f}$ represents the cost incurred when facility $f$ is removed from $S$ ). A list $L$ of best decrease/smallest increase (facility, vertex) pairs is maintained and the actual pair to be swapped is uniform randomly selected from this list. Function Find Pair has the potential of being $\mathcal{O}\left(n^{2}\right)$ however, in practice, this is mitigated as, while $k$ may be large for the initial randomly generated solutions, it rapidly becomes relatively small. In addition, for large problems, a fixed limiting value can be placed on $k$ so that function Find_Pair becomes $\mathcal{O}(n)$. A further optimization that was made is to replace the invocation of procedure Remove_Facility with a simple memory copy of relevant data structures saved prior to the invocation of function Find_Pair.

\section{Empirical Performance Results}

In order to evaluate the performance and behavior of PBS, computational experiments were performed on a benchmark of PC instances (in this context, a PC instance is defined as the combination of a graph $G$ and a particular $p$ value). This benchmark consisted of 
40 instances from the ORLIB ${ }^{1}$ class (Beasley, 1985) and 98 instances from the TSPLIB ${ }^{2}$ class (Reinelt, 1991) giving a total of 138 instances having between 100 and 3038 vertices with $p$ in the range $5, \ldots, 500$.

All experiments for this study were performed on AMD Opteron $2522.6 \mathrm{GHz}$ computer processors that, when executing the DIMACS Machine Benchmark (dmclique ${ }^{3}$ ) on a single processor, required 0.31 CPU seconds for $\mathrm{r} 300.5,1.93 \mathrm{CPU}$ seconds for $\mathrm{r} 400.5$, and 7.35 CPU seconds for r500.5. Note that, as PBS is able to execute using a number of computer processors, in the following sections, PBS- $x$ denotes an $x$ computer processor instance of PBS.

In the following sections, results from a series of experiments that were aimed at providing a detailed assessment of the performance of PBS are first presented. Then, additional experimental results that facilitate a more direct comparison between PBS and VNS (Mladenović et al., 2003) are reported.

\subsection{PBS Performance}

To evaluate the performance of PBS on the instances identified above, extended trials were first performed on those instances where an optimal $p$-center cost was unknown to determine if PBS could improve on the "best known cost" reported to date in the literature. Then, some number (10 or 100) independent trials of PBS were performed for each instance, using a target $p$-center cost identical to the respective optimal or best known cost. The results from these experiments are displayed in Tables 1 and 2 along with the results obtained in previous studies for the exact algorithms ELP (Elloumi et al., 2004), IP* (Al-Khedhairi and Salhi, 2005), Daskin* (Al-Khedhairi and Salhi, 2005), and the meta-heuristic VNS (Mladenović et al., 2003). As PBS uses floating point data and the exact algorithms that it is compared with use integer data, a result obtained by PBS is deemed to be identical to the result obtained by an exact algorithm if the rounded PBS result equals the exact algorithms result. It should be noted that, for PBS, the CPU times do not include the initial reading of input data files nor the creation of static data structures. In addition, for any trial in which PBS did not attain the target $p$-center cost, the reported CPU/elapsed time recorded is that at which the minimum $p$-center cost was attained.

In summary, PBS-1 finds optimal solutions with a success rate of $100 \%$ over 100 trials (that is, all trials were successful) for all instances of the ORLIB class (Table 1) and the small TSPLIB instances (pr226, pr264, pr299, pr439, pcb442, kroA200, kroB200, lin318, gr202, d493, and d657). For the $u 1060$ and $u 1817$ classes (Table 2), with 15 instances each, PBS-1 found all optimal solutions with a success rate of $100 \%$ over 10 trials, produced five new, best known results for $u 1817$, but failed to obtain one currently best known $u 1817$ result. For the 10 instances of the rl1323 class, the PBS- 1 success rate was $100 \%$ over 10 trials for six instances, $80 \%$ with an average percentage deviation of $0.11 \%$ for three instances, and failed on the remaining $r l 1323$ instance with an average percentage deviation of $0.34 \%$. For the pcb3038 class (Table 2), with 14 instances, PBS-16 found 14 new best known costs. These results, over a range of instances, vertices, and facilities, support the claim that PBS is robust and also capable of obtaining high quality results.

\footnotetext{
${ }^{1}$ http: //people.brunel.ac.uk/ mastjjb/jeb/info.html

${ }^{2}$ http: //www.iwr. uni-heidelberg.de/groups/comopt/software/TSPLIB95/

${ }^{3}$ ftp: //dimacs.rutgers. eduindirectory/pub/dsj/clique
} 


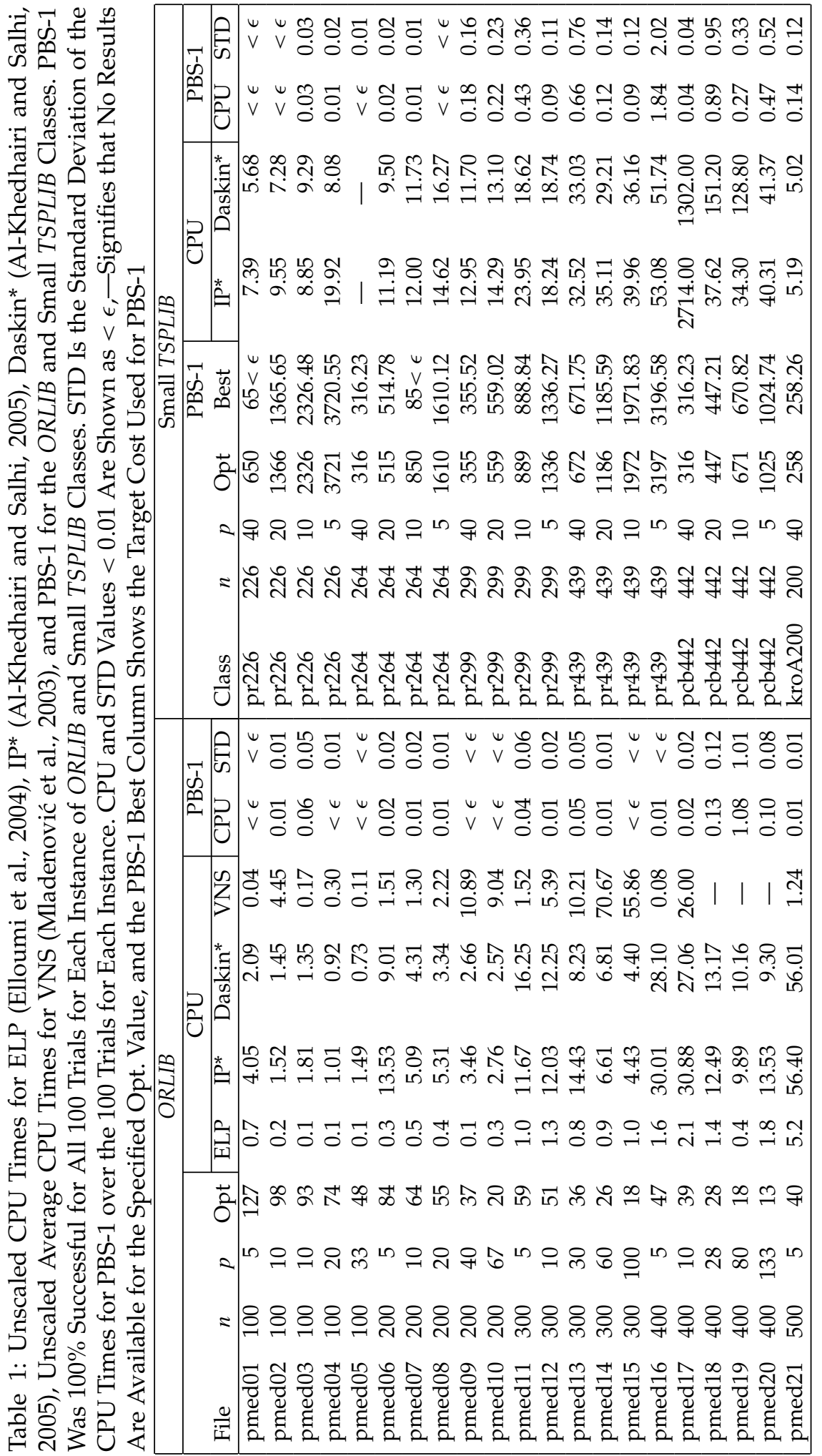




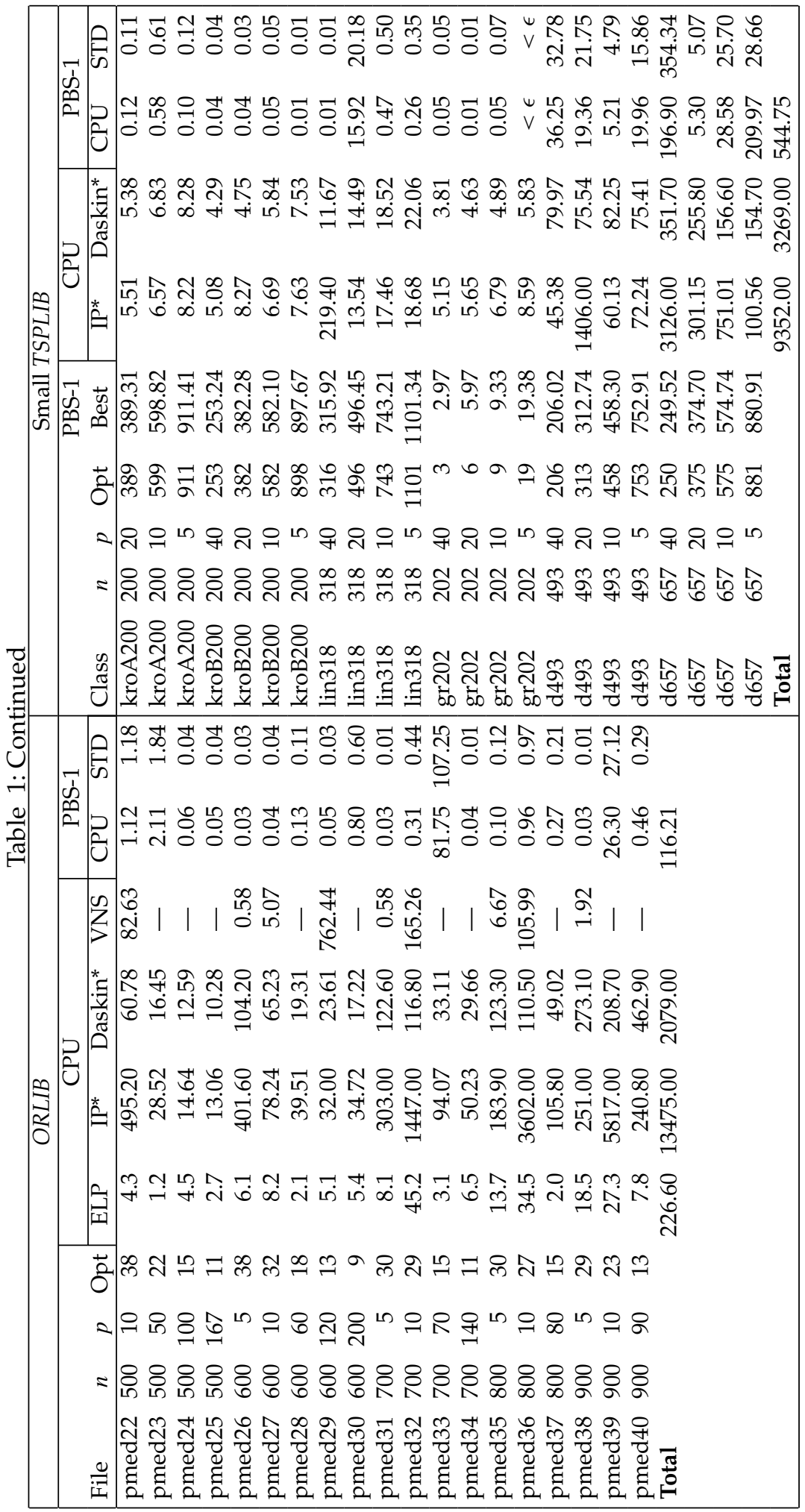




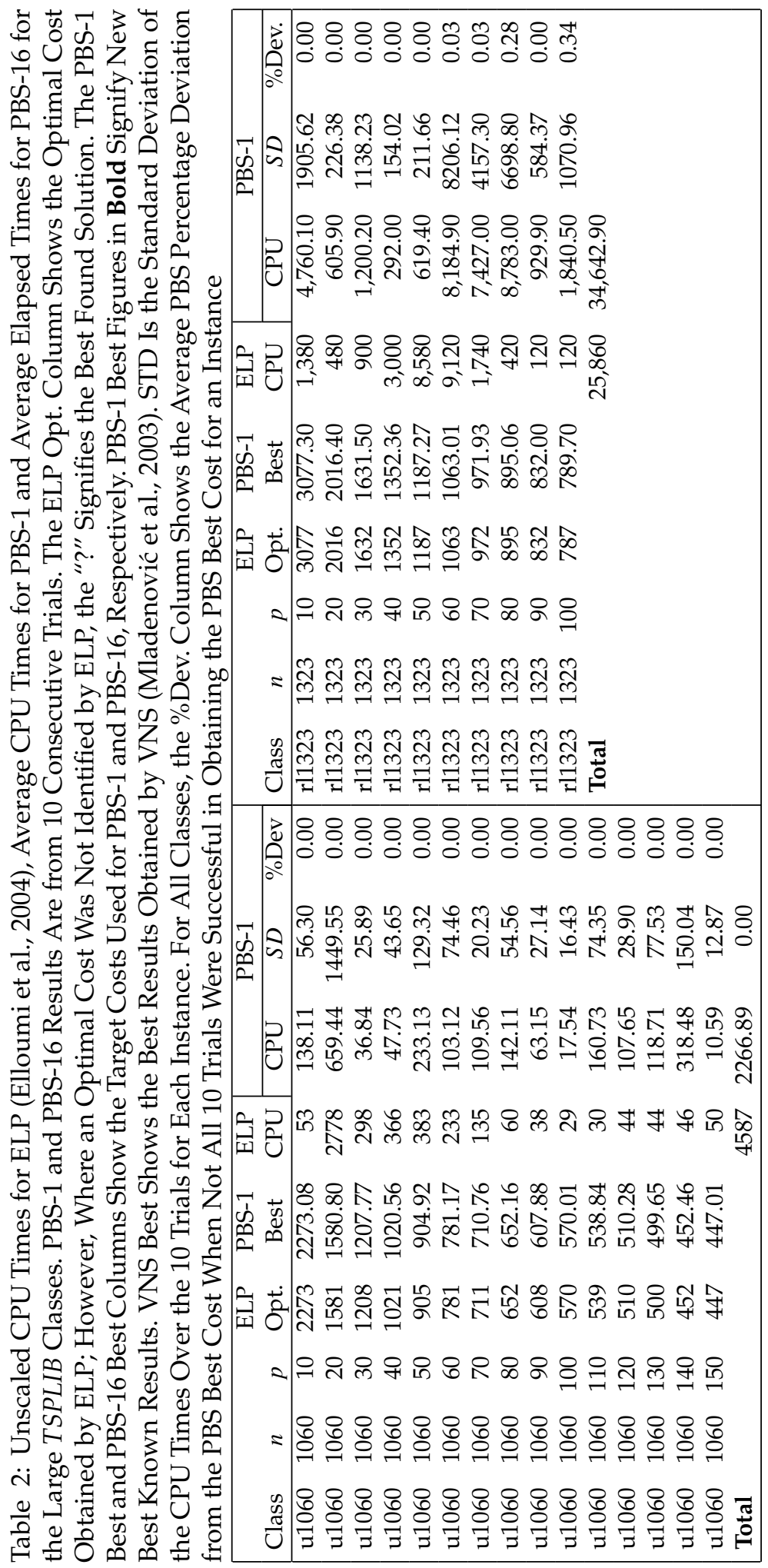




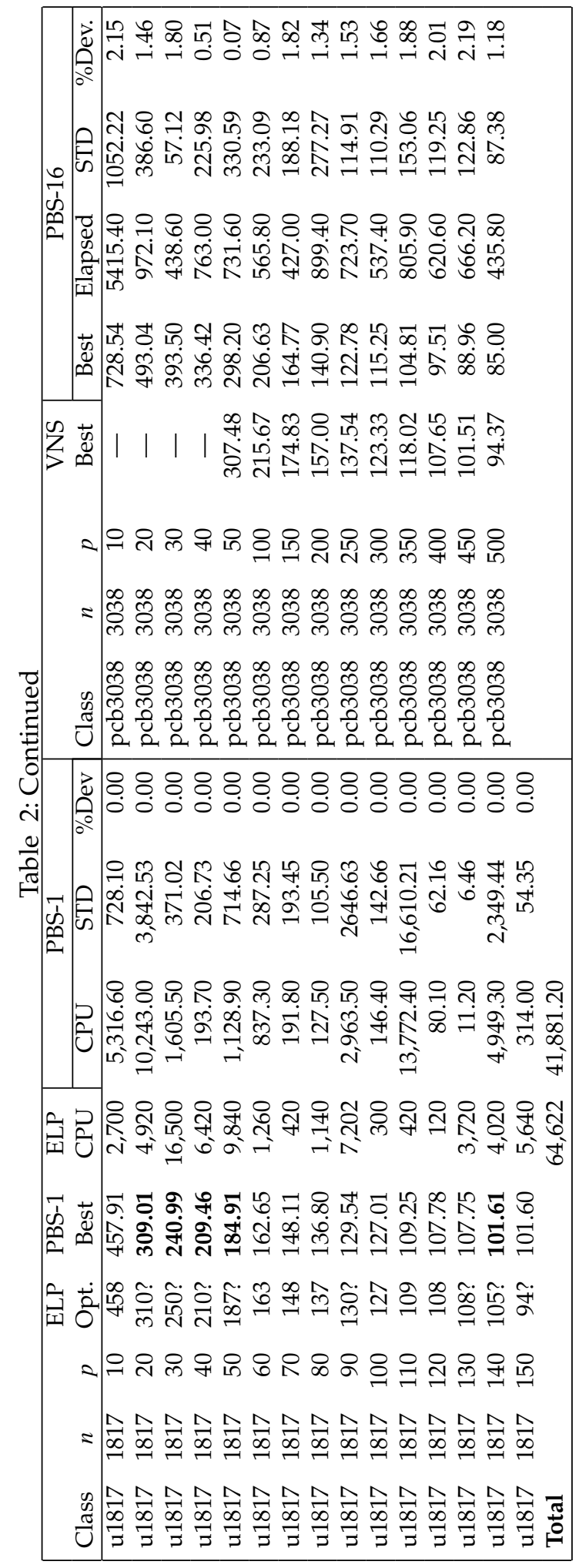




\subsection{Comparative Results}

The results reported in the previous section demonstrate that PBS achieves excellent performance on standard benchmark instances. However, a comparative analysis of these results, as compared to the results found in the literature for other state-of-the-art heuristic PC algorithms, is not a straightforward task as comparisons must take into account the two related metrics of the computational requirements of the algorithms and also the quality of the results produced.

The computational requirements of PBS have been documented in terms of the CPU time/elapsed time used on a computer processor that has been benchmarked using a publicly available benchmark program. However, in previous studies, computing hardware has basically been documented in terms of CPU type (and, sometimes, CPU clock rate) which only allows a very basic means of comparison (i.e., by scaling based on the computer CPU clock rate which, e.g., takes no account of other features, such as memory caching, memory size, hardware architecture, etc.). In addition, differences in what is actually included in the measured times need to be accounted for. With regard to the previous studies referenced in this article, the computer processor used to obtain the ELP (Elloumi et al., 2004) results shown in Tables 1 and 2 was a $400 \mathrm{MHz}$ Pentium II, for $\mathrm{IP}^{*}$ and Daskin* (Al-Khedhairi and Salhi, 2005) a Sun Enterprise Workstation 450 was utilized while for VNS (Mladenović et al., 2003) a Sun Sparc Station 10 was employed.

With regard to the quality of results metric, it must be noted that seemingly small differences in the average $p$-center costs and average percentage cost deviation may in fact represent major differences in algorithm performance, since (as in many hard combinatorial optimization problems) finding $p$-center solutions with slightly higher costs is typically substantially easier than finding those with minimal costs. For example, for the $u 1060, p=130$ instance, achieving an average cost of 500.14 required an average of 5.60 CPU seconds (Table 5) for PBS-1 while achieving an average cost of 499.65 required, on average, $118.71 \mathrm{CPU}$ seconds (Table 2). Basically, the two metrics of computational requirements and the quality of results are related by a nonlinear relationship which can cause the computational requirements to increase exponentially as the quality of the result improves.

\subsubsection{Comparison with Exact Algorithms}

Given the lack of definition of computer processors, an objective comparison of the CPU requirements of PBS with those of the exact algorithms (ELP, IP*, and Daskin*) is basically not possible, however it can be noted that the ranking of the instances, in terms of CPU requirements, is different for each algorithm. With regard to the quality of results, Table 3 provides a summary, for each of the three exact algorithms and PBS, as to the number of optimal or best known solutions found for the 138 instances of the benchmark used in this study. There is a total of 116 instances for which the optimal result is known while best known results only are available for the remaining 22 instances. PBS obtained 112 of the 116 optimal results with a success rate of $100 \%$, three of the remaining four with a success rate of $80 \%$, and failed completely to locate the one remaining optimal result ( $r l 1323$ with $p=100)$. Of the 22 best known results, PBS alone located 19, both PBS and ELP located two more, and only ELP located the one remaining best known result.

\subsubsection{Comparison with VNS}

To enable a more direct comparison with VNS (Mladenović et al., 2003), additional PBS-1 trials were performed in which the target $p$-center cost was set to those obtained 
Table 3: Summary Counts for the Number of Optimal or Best Known Results Found for the Exact Algorithms and PBS for the 138 Instance Benchmark Used in This Study. Opt. Is Optimal Result Known; BK Is Best Known Result Only; - Signifies That an Algorithm Was Not Applied to That Class

\begin{tabular}{|l|rr|rccc|}
\hline \multirow{2}{*}{ Class } & \multicolumn{3}{|c|}{ Solution } & \multicolumn{4}{c|}{ Solutions found } \\
\cline { 2 - 7 } & Type & Count & ELP & IP* & Daskin* & PBS \\
\hline ORLIB & Opt. & 40 & 40 & 40 & 40 & 40 \\
Small TSPLIB & Opt. & 44 & - & 44 & 44 & 44 \\
u1060 & Opt. & 15 & 15 & - & - & 15 \\
rl1323 & Opt. & 10 & 10 & - & - & 9 \\
u1817 & Opt. & 7 & 7 & - & - & 7 \\
u1817 & BK & 8 & 3 & - & - & 7 \\
pcb3038 & BK & 14 & - & - & - & 14 \\
\hline
\end{tabular}

for VNS. While this technique is inherently biased toward VNS (as all PBS-1 results will be less than or equal to the average obtained for VNS and hence the PBS-1 average will be less than that for VNS) it does reduce the impact of the differences in the quality of results on the comparisons and the CPU related differences become the major issue when comparing results obtained by PBS with those obtained for VNS.

Table 4 shows the results obtained where, for all the ORLIB instances, PBS- 1 was run over 100 trials with a target cost that produced $p$-center costs identical to those reported for VNS. The average overall ratio of the average CPU times required by PBS- 1 to those reported for VNS is 0.0007 . Table 5 shows the results obtained where, for the TSPLIB class 41060 , PBS-1 was run over 100 trials with a target cost set equal to the average VNS cost (Mladenović et al., 2003) for this class. The average overall ratio of the average CPU times required by PBS-1 to those reported for VNS (Mladenović et al., 2003) is 0.0270 for the $u 1060$ instances and 0.1107 for the $p c b 3038$ instances. The average of these three ratios is 0.0461 ; however, it is not possible to determine exactly what proportion of this improvement can be attributed to algorithmic differences rather than differences in computing hardware.

\subsubsection{Conclusion}

While these comparisons with other studies suggest that PBS has excellent CPU performance, no final conclusive statement can be made as to the relative CPU performance of the algorithms compared in this section, as differences in computer processor speed and differences as to what is actually included in the time measurements still prevent any concrete conclusions being drawn. The more complete documentation of computer processor performance presented in this study should allow future researchers to make more meaningful comparisons.

\section{Discussion}

To gain a deeper understanding of the runtime behavior of PBS and the efficacy of its underlying mechanisms, additional empirical analyses were performed. Specifically, the variability in runtime between multiple independent runs of PBS on the same problem instance; the effect of adding additional computer processors; the efficiency of the genetic operators; and the effectiveness of the local search were investigated. 
Table 4: PBS-1 Results, from 100 Trials, All of Which Were Successful, and VNS (Mladenović et al., 2003) Results, for the Class ORLIB: Target Cost for PBS-1 is That Reported as Average Cost for VNS; Avg. CPU Is Average CPU Time in Seconds, SD Is the Standard Deviation of the CPU Times. CPU and $S D$ Values $<0.01$ Are Shown as $<\epsilon$

\begin{tabular}{|c|c|c|c|c|c|c|c|c|c|c|c|c|c|}
\hline \multirow[b]{2}{*}{ File } & \multirow[b]{2}{*}{$n$} & \multirow[b]{2}{*}{$p$} & \multirow{2}{*}{$\begin{array}{c}\text { Target } \\
\text { cost }\end{array}$} & \multirow{2}{*}{$\begin{array}{l}\mathrm{VNS} \\
\mathrm{CPU}\end{array}$} & \multicolumn{2}{|c|}{ PBS-1 } & \multirow[b]{2}{*}{ File } & \multirow[b]{2}{*}{$n$} & \multirow[b]{2}{*}{$p$} & \multirow{2}{*}{$\begin{array}{c}\text { Target } \\
\text { cost }\end{array}$} & \multirow{2}{*}{$\begin{array}{l}\text { VNS } \\
\text { CPU }\end{array}$} & \multicolumn{2}{|c|}{ PBS-1 } \\
\hline & & & & & CPU & $S D$ & & & & & & CPU & $S D$ \\
\hline pmed01 & 100 & 5 & 127 & 0.04 & $<\epsilon$ & $<\epsilon$ & pmed21 & 500 & 5 & 40 & 1.24 & 0.02 & 0.01 \\
\hline & 100 & 10 & 98 & 4.45 & 0.01 & 0.01 & ned22 & 500 & 10 & & 82.63 & 1.23 & 1.31 \\
\hline 03 & 100 & 10 & 93 & 0.03 & 0.06 & 0.06 & 123 & 500 & 50 & 3 & 112.33 & 0.02 & 0.01 \\
\hline & 100 & 20 & 74 & 0.30 & $<\epsilon$ & 0.01 & 24 & 500 & 100 & 16 & 264.46 & 0.02 & 0.01 \\
\hline 105 & 100 & 33 & 48 & 0.11 & $<\epsilon$ & $<\epsilon$ & 5 & 500 & 167 & 12 & 175.67 & 0.01 & 0.01 \\
\hline & 200 & 5 & 84 & 1.51 & 0.02 & 0.02 & 26 & 600 & 5 & 38 & 0.58 & 0.04 & 0.03 \\
\hline & 200 & 10 & 64 & 1.30 & 0.01 & 0.02 & 27 & 600 & 10 & 32 & 5.07 & 0.04 & 0.04 \\
\hline & 200 & 20 & 55 & 2.22 & 0.01 & 0.02 & $\operatorname{med} 28$ & 600 & 60 & 19 & 25.09 & 0.02 & 0.01 \\
\hline led09 & 200 & 40 & 37 & 10.89 & $<\epsilon$ & $<\epsilon$ & med29 & 600 & 120 & 13 & 762.44 & 0.05 & 0.03 \\
\hline ned10 & 200 & 67 & 20 & 9.04 & $<\epsilon$ & $<\epsilon$ & pmed30 & 600 & 200 & 11 & 196.95 & 0.02 & $<\epsilon$ \\
\hline hed11 & 300 & 5 & 59 & 1.52 & 0.04 & 0.06 & pme & 700 & 5 & 30 & 0.58 & 0.03 & 0.01 \\
\hline ed12 & 300 & 10 & 51 & 5.39 & 0.01 & 0.02 & pme & 700 & 10 & 29 & 165.26 & 0.32 & 0.45 \\
\hline ed13 & 300 & 30 & 36 & 10.21 & 0.05 & 0.05 & pmed33 & 700 & 70 & 16 & 806.77 & 0.04 & 0.01 \\
\hline ed14 & 300 & 60 & 26 & 70.67 & 0.01 & 0.01 & pmed34 & 700 & 140 & 12 & 160.15 & 0.03 & $<\epsilon$ \\
\hline ed15 & 300 & 100 & 18 & 55.86 & $<\epsilon$ & $<\epsilon$ & pmed35 & 800 & 5 & 30 & 6.67 & 0.10 & 0.12 \\
\hline ed16 & 400 & 5 & 47 & 0.08 & 0.01 & 0.01 & pmed36 & 800 & 10 & 27 & 105.99 & 1.02 & 1.04 \\
\hline d17 & 400 & 10 & 39 & 26.00 & 0.02 & 0.02 & pmed37 & 800 & 80 & 16 & 1197.86 & 0.04 & 0.01 \\
\hline & 400 & 28 & 28 & 119.61 & 0.14 & 0.12 & pmed38 & 900 & 5 & 29 & 1.92 & 0.03 & 0.01 \\
\hline & 400 & 80 & 19 & 300.11 & 0.01 & $<\epsilon$ & pme & 900 & 10 & 24 & 5.98 & 0.05 & 0.03 \\
\hline pmed20 & 400 & 133 & 14 & 218.61 & 0.01 & $<\epsilon$ & pmed 40 & 900 & 90 & 14 & 493.79 & 0.40 & 0.25 \\
\hline & & & & & & & & & & & 5409.38 & 3.94 & \\
\hline
\end{tabular}

Table 5: CPU Averages for PBS-1 (from 100 Trials, All of Which Were Successful) and VNS (Mladenović et al., 2003) for the TSPLIB Instances $u 1060$ and pcb3038. Target Cost for PBS-1 Is That Reported as Average Cost for VNS. Avg. CPU is Average CPU Time in Seconds, SD Is the Standard Deviation of the CPU Times. The \%Dev. Column Shows the Average PBS Percentage Deviation from the PBS Target Cost

\begin{tabular}{|c|c|c|c|c|c|c|c|c|c|c|c|c|}
\hline \multirow[b]{2}{*}{ File } & \multirow{2}{*}{\multicolumn{2}{|c|}{$\begin{array}{c}\text { Target } \\
\text { cost }\end{array}$}} & \multirow{2}{*}{$\begin{array}{l}\text { VNS } \\
\text { CPU }\end{array}$} & \multicolumn{3}{|c|}{ PBS-1 } & \multirow[b]{2}{*}{ File } & \multicolumn{2}{|c|}{ Target VNS } & \multicolumn{3}{|c|}{ PBS-1 } \\
\hline & & & & $\mathrm{CPU}$ & $S D$ & $\%$ Dev & & cost & CPU & $\mathrm{CPU}$ & $S D$ & $\% \mathrm{Dev}$ \\
\hline u1060 & 102 & 2280.09 & 94.93 & 7.89 & 5.29 & 0.00 & pcb3038 & 50317.00 & 578.81 & 66.10 & 69.93 & -0.27 \\
\hline ג1060 & 201 & 1611.95 & 20.49 & 21.79 & 13.71 & -3.19 & pcb3038 & 100220.06 & 570.60 & 61.03 & 46.25 & -0.05 \\
\hline ג1060 & 301 & 1220.41 & 373.46 & 9.25 & 9.87 & -1.28 & pcb3038 & 150174.83 & 52.99 & 460.53 & 289.34 & -0.05 \\
\hline a1060 & 40 & 150.45 & 279.75 & 20.64 & 31.50 & -3.55 & pcb3038 & 200157.88 & 747.00 & 9.40 & 9.82 & -0.14 \\
\hline ג1060 & 50 & 922.14 & 477.18 & 10.46 & 14.36 & -0.07 & pcb3038 & 250140.98 & 103.72 & 4.30 & 3.71 & -0.07 \\
\hline ג1060 & 60 & 806.52 & 446.89 & 4.30 & 5.23 & -0.17 & pcb3038 & 300123.33 & 786.96 & 6.01 & 3.16 & -0.03 \\
\hline 1060 & 70 & 721.37 & 422.73 & 16.86 & 17.14 & 0.00 & pcb3 & 350118.02 & 264.59 & 1.88 & 1.51 & 0.00 \\
\hline 1060 & 80 & 670.53 & 398.84 & 18.10 & 23.84 & -1.45 & & 400107.65 & 904.88 & 5.87 & 2.53 & -0.08 \\
\hline ג1060 & 90 & 640.23 & 111.08 & 3.34 & 2.10 & -0.01 & & 450101.51 & 674.55 & 3.16 & 1.31 & -0.11 \\
\hline ג1060 & 100 & 582.92 & 430.33 & 1.96 & 1.72 & 0.00 & 3038 & $500 \quad 94.37$ & 937.70 & 4.07 & 2.96 & -0.01 \\
\hline u106 & 10 & 565.72 & 186.60 & 1.47 & 1.00 & -0.01 & Total & & 5621.80 & 622.35 & & \\
\hline $\mathrm{u} 106$ & 120 & 551.90 & 218.84 & 0.83 & 0.70 & 0.00 & & & & & & \\
\hline 106 & 130 & 500.14 & 473.65 & 5.60 & 5.13 & 0.00 & & & & & & \\
\hline 106 & 140 & 500.12 & 215.06 & 0.24 & 0.21 & 0.00 & & & & & & \\
\hline u1060 & 150 & 453.16 & 428.16 & 1.02 & 0.80 & 0.00 & & & & & & \\
\hline Total & & & 4577.99 & 123.75 & & & & & & & & \\
\hline
\end{tabular}



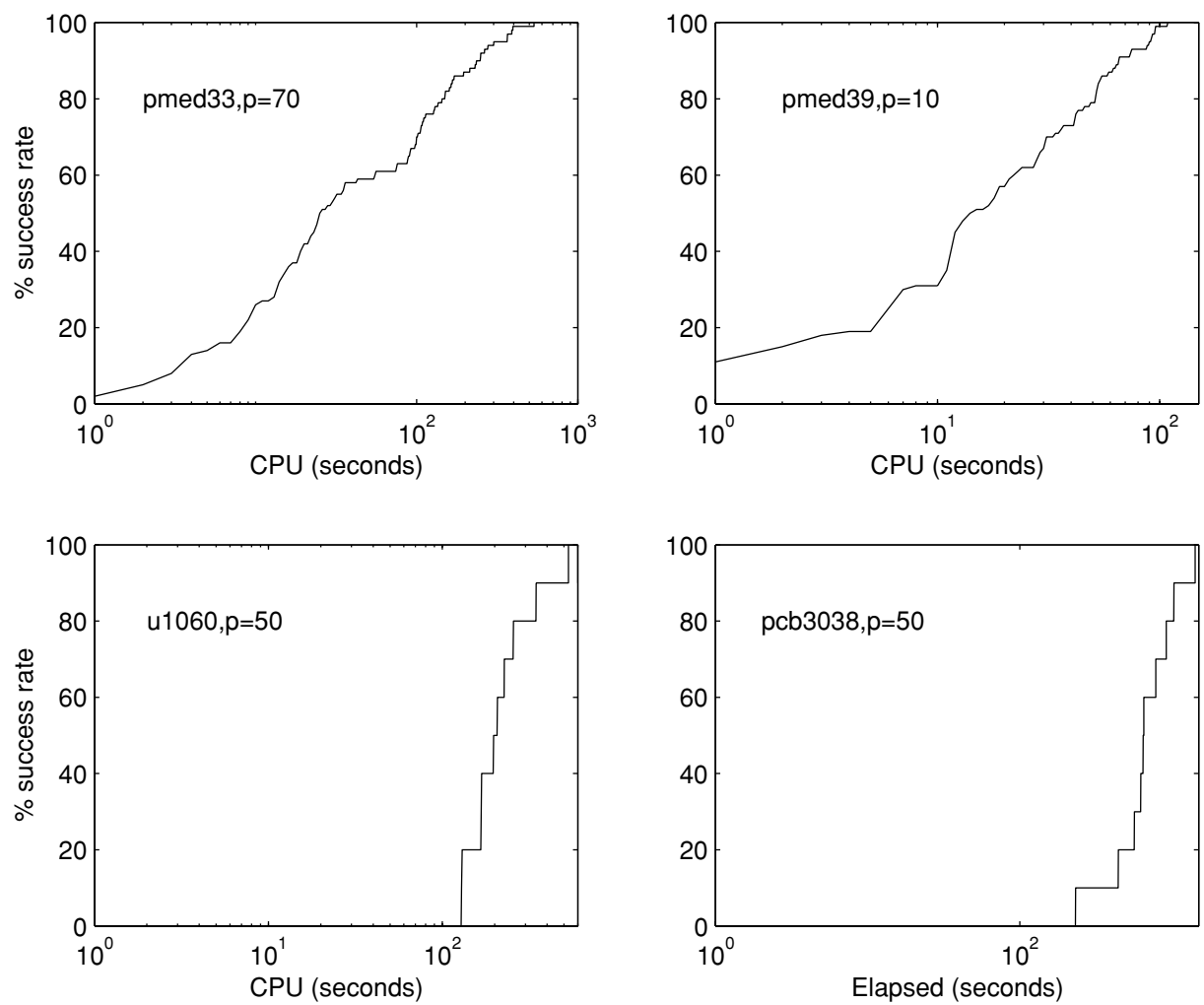

Figure 1: Runtime distributions of CPU time for PBS-1 for 100 trials (all successful) on the pmed33, $p=70$, target $p$-center cost $=15$, pmed39, $p=10$, target $p$-center cost $=23$, and 10 trials (all successful) for both $u 1060, p=50$ and $p c b 3038, p=50$.

\subsection{Variability in Runtime}

The variability of runtime between multiple independent trials of PBS for a particular instance is an important aspect of the behavior of stochastic algorithms. Following the methodology of Hoos and Stützle (2004), this aspect is presented based on runtime distributions (RTDs) of PBS-1 on four problem instances. As can be seen from the empirical RTD graphs shown in Figure 1, PBS-1 shows a reasonably large variability in runtime which is consistent with similar results for other high-performance stochastic search algorithms, for example, for maximum clique (Pullan and Hoos, 2006) and scheduling problems (Watson et al., 2005). Similar observations were made for PBS with regard to the other PC benchmark instances used in this study.

\subsection{Computer Processor Scalability of PBS}

The effect of adding computer processors for PBS to utilize is shown in Table 6 where the median elapsed time for 10 consecutive successful trials for the instance $u 1060$, $p=50$ and target $p$-center cost $=904.92$ is shown. For PBS-x, all $\mathcal{C}, \mathcal{X}_{1}, \mathcal{X}_{2}, \mathcal{M}_{1}, \mathcal{M}_{2}$, and $\mathcal{L}$ operations are performed in parallel in the slave tasks thus providing an $x$-way overlapping of the mutation, crossover, and local search portions of the PBS algorithm. 
Table 6: Effect of the Number of Processors on the PBS- $x$ Median Elapsed Time, Over 10 Successful Trials, for the Instance $u 1060$ with $p=50$, Target $p$-center Cost $=904.92$

\begin{tabular}{|lc|}
\hline$x$ (Number of slave processors) & Median elapsed time \\
\hline 1 & 196.00 \\
2 & 101.00 \\
3 & 52.00 \\
4 & 16.00 \\
\hline
\end{tabular}

Table 7: Percentage Frequency of the Local Search, During the Initial Creation of $P$ and After Each Genetic Operator, Obtaining the Target $p$-center Cost for All Instances of the ORLIB $, d 493, d 657, u 1060, r l 1323$, and $u 1817$ Classes

\begin{tabular}{|lrrrrrr|}
\hline $\mathcal{L}$ Invocation & $\begin{array}{r}\text { ORLIB } \\
\text { \%Freq. }\end{array}$ & $\begin{array}{c}d 493 \\
\text { \%Freq. }\end{array}$ & $\begin{array}{r}\text { \%657 } \\
\text { \%Freq. }\end{array}$ & $\begin{array}{r}u 1060 \\
\text { \%Freq. }\end{array}$ & $\begin{array}{r}\text { rl1323 } \\
\text { \%Freq. }\end{array}$ & $\begin{array}{r}u 1817 \\
\text { \%Freq. }\end{array}$ \\
\hline During creation of $P$ & 98.9 & 71.9 & 52.8 & 42.5 & 4.3 & 39.3 \\
$\mathcal{M}_{1}$ & 0.1 & 3.0 & 2.8 & 6.4 & 7.4 & 3.3 \\
$\mathcal{M}_{2}$ (after $\mathcal{M}_{1}$ ) & 0.2 & 4.3 & 10.9 & 2.8 & 4.3 & 4.7 \\
$\mathcal{M}_{2}\left(\right.$ after $\mathcal{X}_{1}$ ) & 0.2 & 4.3 & 5.0 & 6.4 & 8.5 & 6.0 \\
$\mathcal{M}_{2}$ (after $\mathcal{X}_{2}$ ) & 0.4 & 4.5 & 17.7 & 12.8 & 17.0 & 24.0 \\
$\mathcal{X}_{1}$ (before $\mathcal{M}_{2}$ ) & 0.1 & 4.5 & 2.8 & 7.8 & 11.7 & 8.0 \\
$\mathcal{X}_{2}$ (before $\mathcal{M}_{2}$ ) & 0.1 & 7.5 & 8.0 & 21.3 & 46.8 & 14.7 \\
\hline
\end{tabular}

\subsection{Effectiveness of PBS Components}

Table 7 gives the frequency with which the local search $(\mathcal{L})$ located the target cost after being invoked during the initial creation of the population $P$ and after each of the genetic operators for all instances of the ORLIB, $d 493, d 657, u 1060, r l 1323$, and $u 1817$ classes. The effectiveness of the directed mutation $\left(\mathcal{M}_{2}\right)$ and phenotype $\left(\mathcal{X}_{2}\right)$ genetic operators, as compared to the random versions, for the more difficult classes is highlighted by the results shown in Table 7 where, for the $u 1060$ class, $\mathcal{M}_{2}$ and $\mathcal{X}_{2}$ accounted for $43.3 \%$ of target cost locations (as compared to a total of $14.2 \%$ for $\mathcal{M}_{1}$ and $\mathcal{X}_{1}$ ), for rl1323, $76.6 \%$ of target cost locations $\left(19.1 \%\right.$ for $\mathcal{M}_{1}$ and $\left.\mathcal{X}_{1}\right)$ and for $u 1817,49.4 \%$ of target cost locations $\left(11.3 \%\right.$ for $\mathcal{M}_{1}$ and $\left.\mathcal{X}_{1}\right)$.

Figure 2 gives more detailed information on the overall performance of the two genetic crossover operators in that it shows the evolution, for each application of each crossover operator, of the average $p$-center cost for the parents to the actual $p$-center cost of the child solutions after the genetic operator but prior to the local search. The effectiveness of the genetic operators in generating a wide range of starting points for the local search, from a population of eight solutions, is clearly demonstrated by the wide range of child $p$-center costs. The horizontal bands in the plots are caused by repeated use of the same parent $p$-center solutions from $P$. Of interest is that $\mathcal{X}_{2}$, on average, generates child $p$-center solutions of lower cost than those generated by $\mathcal{X}_{1}$ which illustrates the effectiveness of phenotype crossover operators for problems such as $p$-center.

\subsection{Local Search}

Figures 3 and 4 show, for the pmed23, pmed33, u1060, and $u 1817$ instances, the average cost of the $p$-center solution at each iteration of the PBS local search. In all cases, there is an initial fast decrease followed by an extended search over a relatively flat 

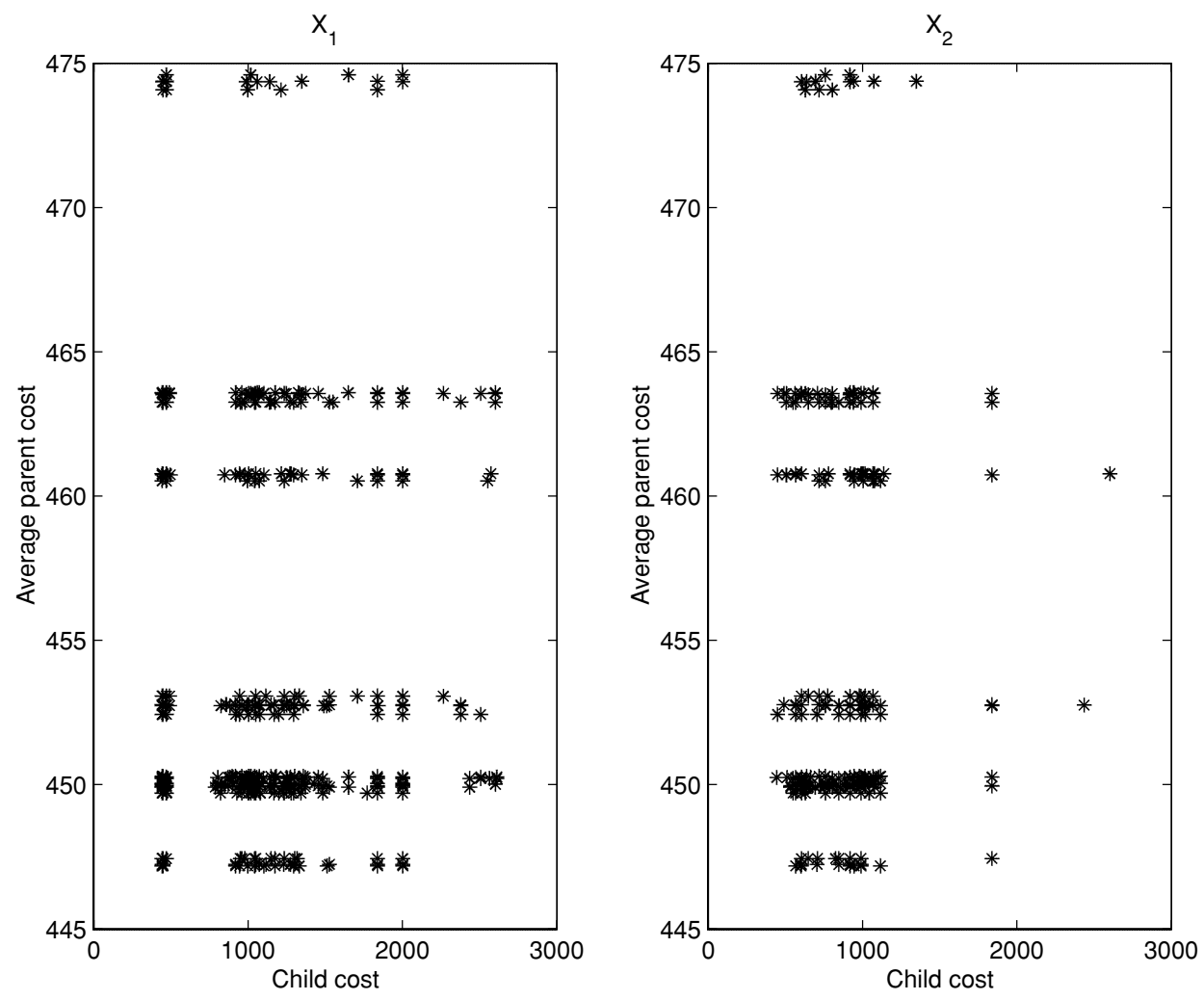

Figure 2: For the $u 1060$ instance, cost (prior to the local search) of the child $p$-center solutions generated by $\mathcal{X}_{1}$ and $\mathcal{X}_{2}$ as compared to the average parent cost.

cost hypersurface. For pmed23 and pmed33, as p-center cost is an integer value, a large proportion of the cost hypersurface appears to consist of flat areas with \pm 1 local maxima/minima. For $u 1060$ and $u 1817$, p-center cost is a real value which gives rise to a cost hypersurface with few actual flat areas but a very large number of local minima. Clearly, the acceptance of non-improving facility-vertex swaps and preventing the re-use of facility-vertex swaps within a single invocation of local search are important features of the PBS local search in dealing with these types of hypersurfaces.

Also shown in Figures 3 and 4 is the actual progress of local search for the successful invocation of local search which, in all cases, started from a relatively low cost $p$-center solution. This indicates that the PBS meta-heuristic of generating new starting points for local search based on existing low cost $p$-center solutions (via the $\mathcal{M}_{1}, \mathcal{M}_{2}, \mathcal{X}_{1}, \mathcal{X}_{2}$ genetic operators) is an effective approach for the $p$-center problem.

Finally, Figures 3 and 4 show the percentage of invocations that reached each iteration level of local search. The effect of the local search termination condition $0.1(g+1) n$ in ensuring that a reasonable search around the starting point is performed before termination is evident.

\section{Conclusions and Future Work}

This study has demonstrated that by applying the general paradigm of using local search, within a population based meta-heuristic incorporating directed mutation and 

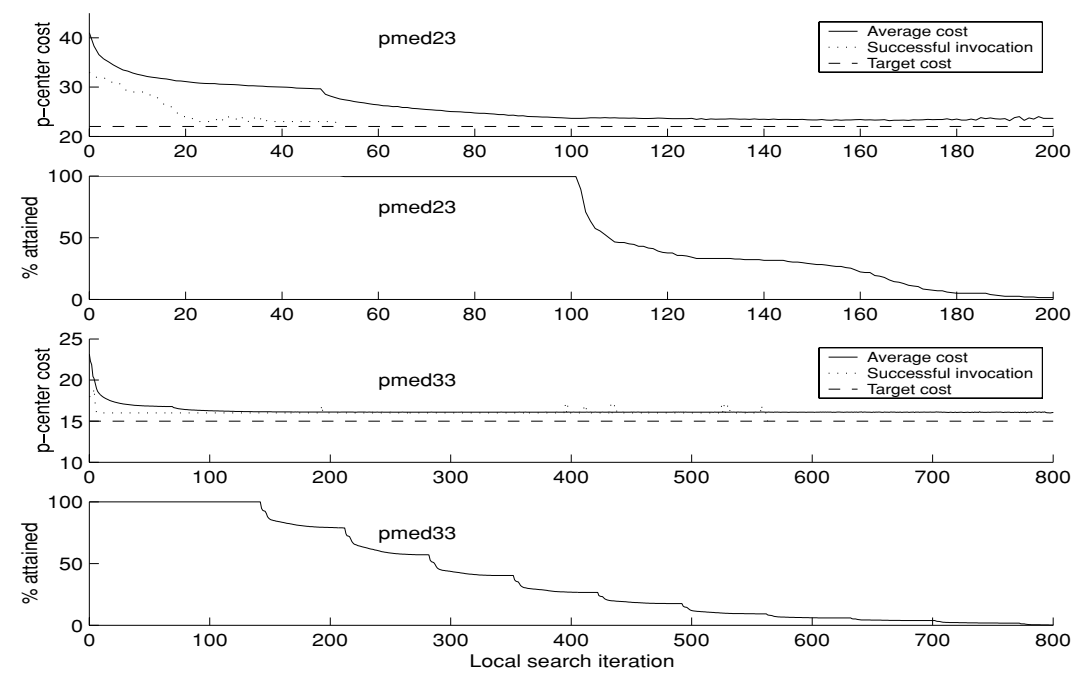

Figure 3: For the pmed23 and pmed33 instances, average cost of the $p$-center solution at each iteration of the PBS local search. Also shown are the actual progress of local search for the successful invocations which, in both cases, started from a relatively low cost $p$-center solution and the frequency with which each invocation of local search reached a given number of iterations.
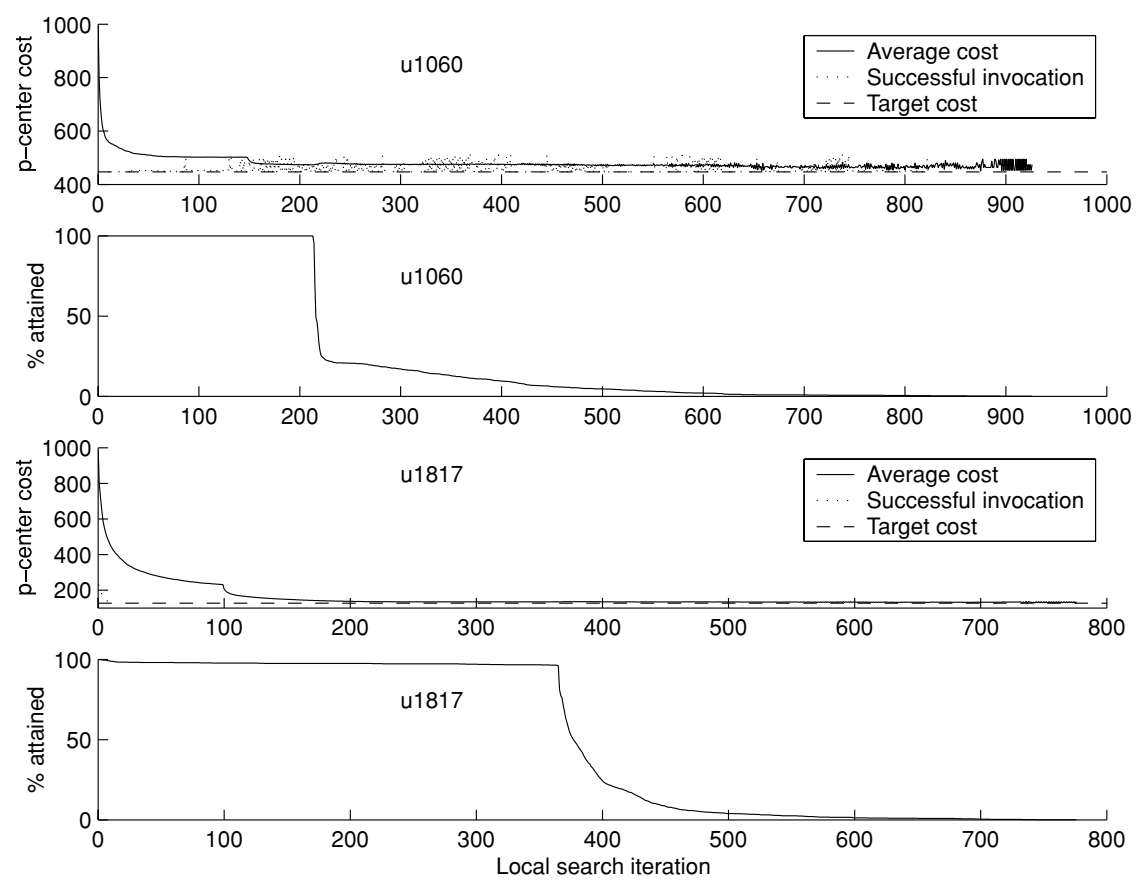

Figure 4: For the $u 1060$ and $u 1817$ instances, average cost of the $p$-center solution at each iteration of the PBS local search. Also shown are the actual progress of local search for the successful invocations which, in both cases, started from a relatively low cost $p$-center solution and the frequency with which each invocation of local search reached a given number of iterations. 
phenotype crossover genetic operators an effective meta-heuristic for the $p$-center problem can be created. In summary, with regard to the robustness and quality of the PBS results, for the 138 benchmark instances, for the 116 instances where the optimal solution is known, PBS located 112 of these with a success rate of $100 \%$. For the 22 instances where the optimal solution is not known, PBS located 19 new best known solutions and two currently best known solutions. With regard to computational performance, while the comparisons performed with the only other relevant meta-heuristic suggest that PBS has excellent performance, due to difficulties in realistically comparing computational performance with that documented for other algorithms, no final conclusive statement can be made as to the relative CPU performance of PBS as compared to other algorithms.

Given the excellent performance of PBS on the standard PC instances reported here, the algorithm clearly has substantial potential to provide the basis for high-performance algorithms for other clustering combinatorial optimization problems such as capacitated $p$-median, maximum covering, uncapacitated/capacitated facility location, set covering, and weighted maximum satisfiability.

\section{References}

Al-Khedhairi, A. and Salhi, S. (2005). Enhancements to two exact algorithms for solving the vertex p-center problem. Journal of Mathematical Modelling and Algorithms, 4:129-147.

Beasley, J. E. (1985). A note on solving large p-median problems. European Journal of Operational Research, 21:270-273.

Cheng, T., Kang, L., and Ng, C. (2007). An improved algorithm for the $p$-center problem on interval graphs with unit lengths. Computers and Operations Research, 4(8):2215-2222.

Cranic, T., Gendreau, M., Hansen, P., and Mladenović, N. (2004). Cooperative parallel variable neighbourhood search for the p-median. Journal of Heuristics, 10:293-314.

Daskin, M. (1995). Network and Discrete Locations: Models, Algorithms and Applications. Wiley, New York.

Daskin, M. (2000). A new approach to solving the vertex p-center problem to optimality: Algorithm and computational results. Communications of the Operations Research Society of Japan, 45:428-436.

Elloumi, S., Labbe, M., and Pochet, Y. (2004). A new formulation and resolution method for the p-center problem. INFORMS Journal on Computing, 16:84-94.

Hansen, P., Mladenović, N., and Perez-Brito, D. (2001). Variable neighborhood decomposition search. Journal of Heuristics, 7:335-350.

Hassin, R., Levin, A., and Morad, D. (2003). Lexicographic local search and the $p$-center problem. European Journal of Operational Research, 151:265-279.

Hochbaum, D. and Shmoys, D. (1985). A best possible heuristic for the $k$-center problem. Mathematical Operations Research, 10:180-184.

Hoos, H. H. and Stützle, T. (2004). Stochastic Local Search: Foundations and Applications. Morgan Kaufmann, San Mateo, CA.

Ilhan, T. and Pınar, M. C. (2001). An efficient exact algorithm for the vertex $p$-center problem. Online report, http://www.optimization-online.org/db_html/2001/ 09/376.html.

Kariv, O. and Hakimi, L. (1979). An algorithmic approach to nework location problems, Part ii, The $p$-medians. SIAM Journal of Applied Mathematics, 37:539-560. 
Krumke, S. (1995). On a generalization of the p-center problem. Information Processing Letters, 56(2):67-71.

Martinich, J. (1988). A vertex-closing approach to the $p$-center problem. Naval Res. Log., 35:185201.

Minieka, E. (1970). The $m$-center problem. SIAM Review, 12:138-139.

Mladenović, N., Labbe, M., and Hansen, P. (2003). Solving the $p$-center problem with tabu search and variable neighbourhood search. Networks, 42:48-64.

Plesnik, J. (1987). A heuristic for the p-center problem in graphs. Discrete Applied Mathematics, 17:263-268.

Pullan, W. (2005). An unbiased population-based search for the geometry optimization of Lennard-Jones clusters: $2 \leq n \leq 372$. Journal of Computational Chemistry, 26:899-906.

Pullan, W. and Hoos, H. (2006). Dynamic local search for the maximum clique problem. Journal of Artificial Intelligence Research, 26:159-185.

Reinelt, G. (1991). TSPLIB: A traveling salesman problem library. ORSA Journal on Computing, 3:376-384.

Resende, M. and Werneck, R. (2004). A hybrid heuristic for the p-median problem. Journal of Heuristics, 10:59-88.

Scaparra, M., Pallottino, S., and Scutellà, M. (2004). Large-scale local search heuristics for the capacitated vertex $p$-center problem. Networks, 43(4):241-255.

Taillard, E. D. (2003). Heuristic methods for large centroid clustering problems. Journal of Heuristics, 9:51-74.

Tansel, B. C., Francis, R. L., and Lowe, T. J. (1983). Location on networks: A survey. Management Science, 29:482-511.

Watson, J., Whitley, L., and Howe, A. (2005). Linking search space structure, run-time dynamics, and problem difficulty: A step toward demystifying tabu search. Journal of Artificial Intelligence, 24:221-261.

Whitaker, R. (1983). A fast algorithm for the greedy interchange of large-scale clustering and median location problems. INFOR, 21:95-108. 